\title{
Article \\ Molecular Characterization and Functional Analysis of Two Steroidogenic Genes TSPO and SMAD4 in Yellow Catfish
}

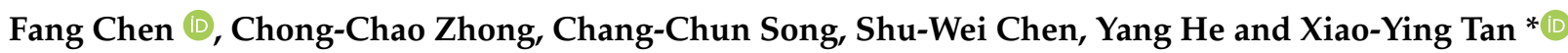 \\ Key Laboratory of Freshwater Animal Breeding, Ministry of Agriculture, Fishery College, Huazhong Agricultural \\ University, Wuhan 430070, China; chenfang95@webmail.hzau.edu.cn (F.C.); \\ zhongchongchao@webmail.hzau.edu.cn (C.-C.Z.); songchangchun@webmail.hzau.edu.cn (C.-C.S.); \\ chenshuwei@webmail.hzau.edu.cn (S.-W.C.); heyang1997@webmail.hzau.edu.cn (Y.H.) \\ * Correspondence: txy7933@mail.hzau.edu.cn; Tel.: +86-27-8728-2113
}

Citation: Chen, F.; Zhong, C.-C.; Song, C.-C.; Chen, S.-W.; He, Y.; Tan, X.-Y. Molecular Characterization and Functional Analysis of Two Steroidogenic Genes TSPO and SMAD4 in Yellow Catfish. Int. J. Mol. Sci. 2021, 22, 4505. https://doi.org/ $10.3390 /$ ijms22094505

Academic Editor: Vladimir Trifonov

Received: 26 March 2021

Accepted: 22 April 2021

Published: 26 April 2021

Publisher's Note: MDPI stays neutral with regard to jurisdictional claims in published maps and institutional affiliations.

Copyright: (c) 2021 by the authors. Licensee MDPI, Basel, Switzerland. This article is an open access article distributed under the terms and conditions of the Creative Commons Attribution (CC BY) license (https:// creativecommons.org/licenses/by/ $4.0 /)$.

\begin{abstract}
The steroid hormones are required for gonadal development in fish. The present study was undertaken to characterize the cDNA and promoter sequences of TSPO and SMAD4 genes in yellow catfish Pelteobagrus fulvidraco, explored the mRNA tissue expression and deciphered their promoter regions. Yellow catfish TSPO and SMAD4 shared the similar domains to the corresponding genes from other vertebrates. The TSPO and SMAD4 mRNAs were widely expressed in the detected tissues, but at different levels. Several transcription factors were predicted, such as Sp, GATA, AP1, SOX1, SRY, STAT, HNF4 $\alpha$, PPAR $\gamma$, Pu.1 and FOXL2. PPAR $\gamma$ overexpression increased but STAT3 overexpression reduced TSPO promoter activity, and FOXL2 overexpression inhibited the promoter activity of TSPO and SMAD4. The site mutation and EMSA analysis indicated that TSPO promoter possessed STAT3 and FOXL2 sites. Overall, our provided the novel understanding into the transcriptionally regulatory mechanisms of TSPO and SMAD4 in fish.
\end{abstract}

Keywords: Pelteobagrus fulvidraco; steroidogenesis-related genes; molecular characterization; promoter; transcriptional regulation

\section{Introduction}

Steroid hormones modulate embryonic development, sex differentiation, metabolism and reproduction in vertebrates. Translocator protein (TSPO), located in the mitochondrial outer membrane, plays the important roles in the transport of cholesterol to the inner mitochondrial membrane, where cholesterol was converted into pregnenolone by cytochrome P450scc (CYP11A1) [1,2]. Mothers against decapentaplegic homolog 4 (SMAD4) acts as a co-regulator and mediates the transcriptional regulation of CYP19A1 [3], and CYP19A1 is responsible for the formation of $\mathrm{C}_{18}$ steroids, which is the most important enzyme in the control of sex development in the fish [4]. Owing to the importance of TSPO and SMAD4 in maintaining the steroidogenesis, it is very essential to study their molecular characterization and the transcriptional regulation. At present, several studies have explored the functional characterizations of TSPO and SMAD4 in several physiological processes [5]. In mammals, the TSPO promoter was cloned and identified from human breast cancer cells, and the binding sites of specificity protein (SP1, SP3 and SP4) positively regulate the activity of TSPO promoter [6]. Similarly, the promoter of SMAD4 was isolated and cloned from patients with thyroid tumor, demonstrating the importance of GC box on the activity of SMAD4 [7]. However, information associated with the molecular characterization and transcriptional regulation of TSPO and SMAD4 promoters were very scarce in fish.

In eukaryotic organisms, promoter regions have many cis-acting elements and regulate the expression of genes at the transcriptional level by binding with transcriptional factors [8]. Therefore, in order to study the regulatory mechanism of TSPO and SMAD4 genes, we should at first explore the structure and function of their promoters. At present, 
several transcriptional factors, such as Sp sites and activator protein-1 (Ap1), were predicted at TSPO and SMAD4 promoters $[9,10]$. In addition, signal transducer and activator of transcription 3 (STAT3), peroxisome proliferator-activated receptor gamma (PPAR $\gamma$ ) and forkhead box 12 (FOXL2) are three important transcription factors that regulate the expression of many target genes involved in numerous physiological processes, including steroidogenesis $[11,12]$. Considering that TSPO and SMAD4 are also the key enzymes and transcription factors in regulating steroidogenesis [1,3], we hypothesized that PPAR $\gamma$, STAT3 and FOXL2 regulated steroidogenesis by targeting TSPO and SMAD4.

Yellow catfish Pelteobagrus fulvidraco, widely distributed freshwater fish, is an important economic fish in some Asian countries due to the high economic value and delicious meat quality. At present, studies were scarce on the regulation of steroidogenesis for the fish species [13]. To this end, we cloned and characterized the cDNA and promoter sequences of TSPO and SMAD4 genes, and determined their mRNA tissue expression and investigated their transcriptional regulation in yellow catfish. Our study provides new understanding into characterizing the role of steroid-related genes during ovarian development.

\section{Results}

\subsection{Molecular Characterization}

The full-length cDNAs of TSPO and SMAD4 genes from P. fulvidraco were $952 \mathrm{bp}$ and $2221 \mathrm{bp}$ in length, respectively (Table 1 ). Their predicted amino acid sequences TSPO and SMAD4 were identical to those from other fish and mammals, and the amino acid sequence identities between TSPO and other species ranged from $39.63 \%$ to $69.12 \%$, and SMAD4 from $63.93 \%$ to $94.19 \%$ (Table 2 ).

Table 1. The sequence information of TSPO and SMAD4 Genes from P. fulvidraco.

\begin{tabular}{ccccccc}
\hline Gene & $\begin{array}{c}\text { Accession } \\
\text { No. }\end{array}$ & $\begin{array}{c}\mathbf{5}^{\prime} \text { UTR } \\
\mathbf{( b p )}\end{array}$ & $\begin{array}{c}\text { ORF } \\
(\mathbf{b p})\end{array}$ & $\begin{array}{c}\mathbf{3}^{\prime} \mathbf{U T R} \\
\mathbf{( b p )}\end{array}$ & $\begin{array}{c}\text { Full Length } \\
(\mathbf{b p})\end{array}$ & $\begin{array}{c}\text { Protein } \\
(\mathbf{a a})\end{array}$ \\
\hline TSPO & MN188059 & 116 & 486 & 350 & 952 & 161 \\
SMAD4 & MN188058 & 202 & 1725 & 294 & 221 & 574 \\
\hline
\end{tabular}

Table 2. Amino acid sequence identity of TSPO and SMAD4 Genes between P. fulvidraco and other species (\%).

\begin{tabular}{cccccc}
\hline Gene & Ictalurus punctatus & Danio rerio & Xenopus tropicalis & Homo sapiens & Rattus norvegicus \\
\hline TSPO & 69.12 & 61.29 & 50.69 & 41.94 & 39.63 \\
SMAD4 & 94.19 & 63.93 & 65.64 & 66.32 & 66.32 \\
\hline
\end{tabular}

Notes: Accession numbers as follows (the order is Ictalurus punctatus, Danio rerio, Xenopus tropicalis, Homo sapiens and Rattus norvegicus): TSPO (XM 017479065.1, NM 001006032.2, XM 012956428.2, NM 001256531.1, NM 012515.2; SMAD4 (XM 017471854.1, EU489481.1, XM 002934439.4, NM 005359.5, NM 019275.3).

The TSPO protein sequence from P. fulvidraco had the five $\alpha$-helix transmembrane structure (TM1-5), the C-terminal cholesterol-recognition amino acid consensus domain (CRAC) (Figure S1). The P. fulvidraco SMAD4 possessed the MH1 domain at the $\mathrm{N}$ terminus, the MH1 domain at the $C$ terminus, the SMAD4 activation domain (SAD), the DNA binding motif, the nuclear localization and export signals (NLS and NES) (Figure S2). 


\subsection{Tissue Distribution of Gene Expression}

TSPO and SMAD4 mRNA expression levels were detected in the tested tissues from P. fulvidraco, but their mRNA levels were variable among the tissues (Figure 1). TSPO mRNA levels were predominant in the spleen, followed by the testis, kidney and muscle. SMAD4 mRNA levels were the highest in the testis, followed by the liver and ovary, and the lowest in the kidney.

TSPO

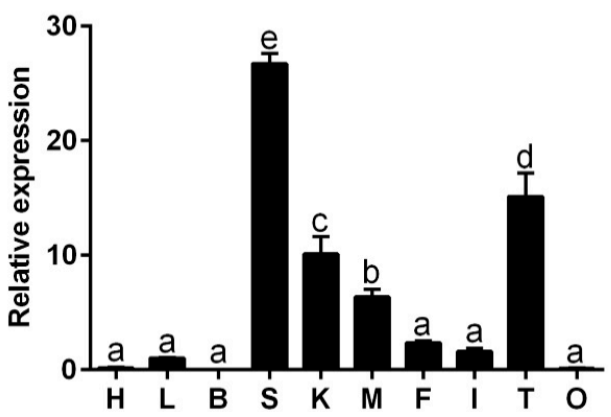

SMAD4

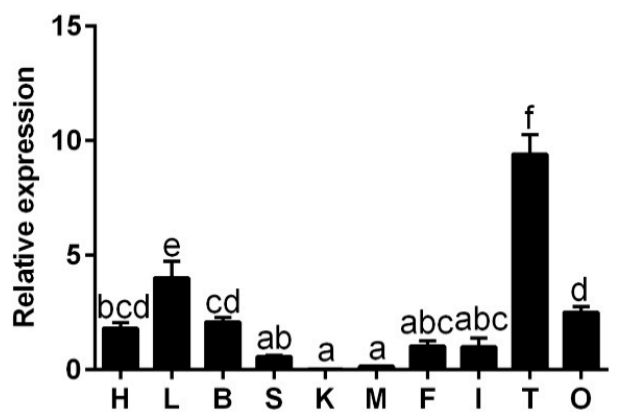

Figure 1. Quantitative PCR (Q-PCR) analysis for mRNA expression of TSPO and SMAD4 across heart $(\mathrm{H})$, liver $(\mathrm{L})$, brain $(\mathrm{B})$, spleen $(\mathrm{S})$, kidney $(\mathrm{K})$, muscle $(\mathrm{M})$, fat $(\mathrm{F})$, intestine (I), testis $(\mathrm{T})$ and ovary (O) of $P$. fulvidraco. Data (mean $\pm \mathrm{SEM}, n=3$ replicates. For each replicate, 4 fish were sampled) were expressed relative to expression of reference gene ( $\beta$-actin and ubce). Bars that share different letters indicate significant differences among various tissues $(p<0.05)$.

\subsection{Sequence Analysis of the Promoter Regions of TSPO and SMAD4}

We obtained the $2015 \mathrm{bp}$ of TSPO and the $1506 \mathrm{bp}$ of SMAD4 promoters. On the TSPO promoter region (Figure 2), three binding sites for Sp factors (Sp1/3) were located in position $+7 \mathrm{bp}$ to $-8 \mathrm{bp},-794 \mathrm{bp}$ to $-803 \mathrm{bp}$, respectively, and $-445 \mathrm{bp}$ to $-455 \mathrm{bp}$, respectively, two AP1 binding sites between $-44 \mathrm{bp}$ and $-55 \mathrm{bp}$, and $-1344 \mathrm{bp}$ and $-1384 \mathrm{bp}$, respectively, and one PPAR $\gamma$ binding site at $-734 \mathrm{bp}$ to $-748 \mathrm{bp}$, and a single putative STAT3 site at the position $-1936 \mathrm{bp}$ to $-1946 \mathrm{bp}$. Other putative binding sites included FOXL2, GATA, HNF4 $\alpha$, SOX1, SRY, STAT1 and Pu.1 at their promoter regions. On the SMAD4 core promoter (Figure 3), a TATA-like box was located from $-83 \mathrm{bp}$ to $-88 \mathrm{bp}$, three Sp1 located from $-34 \mathrm{bp}$ to $-43 \mathrm{bp},-60 \mathrm{bp}$ to $-70 \mathrm{bp}$ and $-72 \mathrm{bp}$ to $-82 \mathrm{bp}$, respectively. Other binding sites included DCE, HNF4 $\alpha$, FOXL2 and AP1 on the promoter region of SMAD4 gene. 
CTTAGCAGAC AGAGTGGGAA TGGTCTGAAA TTTTAaGATA TGCTTCCAGA ATGTATTTAC AGCAGAGCAT TTCCAAAAAA ATGGATTAAT -1926 STAT3

GGAACAATTT TTTTAAATCA CTGCCCCAAT AGTGTAGCTC TAAAGTGATT AACTAGCATA CAAATTAGCA ATGTAATTAG TCTGTTTTCT -1836

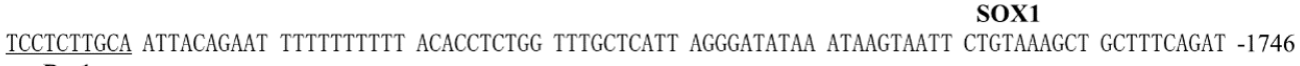
Pu.1

AAAATCTACT GTTAAACTCT ATATGATATC TGGTTTATAT ACTTTGATTA TCAGAACCAA TTACTTGCAA ACAAATGTGC ATGATAAGTA -1656 FOXL2

AaTATGCACA ATAACTGATT TCATTATATT TGgGTGATTA TAGTTTTATG CCAATGAAAA ATAGTATAAT TTACTGACTG TATAATTATA -1566 ACATTATTAC AatCATGTTA GTCTGAGGAC ATTAGGgTtC CCAATTCTTT TTTCAGgCAG GACATTATAT TGTGTAGCAA AGAAAGAATA -1476 GTTTTAACAA AAAAATAAAA AAATAAAATA AACAATTCTA AACTAAACAA AATTTAATTT CAAATGAAAA TTTTAAAGAG AACCAGTACA -1386 SRY

TAATGAATCA CAATGTCACA GTACCTTTCG AGATACTTTG TAATTTGCAC TGTTATTAAT TTAACTGGAa TTTAAAGGAa CCATTGAGTT -1296 AP1

GATGAAGTTT TAATCCAAGG AGCACCAAGT CCCTCAACTG GTCAGTTGTG TAAATGAGAT AGACTTTCAT TAAATTGATT ACATTGTTTT -1206

TTATTGATTG CCCCAATAAT GCACTCACTA CCTCTCAATA ATATTAATAT TTAACAACAT TGCACATTTT GCCATTTTTT ATTTTGTTCA -1116 ATAGAAATCT TAAAAGAGAT AATGTCTAAT TTTTAGGAGC TTTAAATCTG GGAATTCAGT ATAACCTATT TTAAGGACCA TTATATAGTA -1026 TATACAATCT GCAGGTAaCC ATAATGCAGA TTCAAGTAAA CTGACAgTaT CACTGGCTAT GATCTGTGAG GTAGCAGAAG GAGAGGTAGA -936 TCCTGAATAG TGTTACATGg GAAAATAGTT TAAATGCCCA AGGTAGTGAT ATACTATGTG TGTATCTATG GTTATGTATT CATACAAACC -846 AAAGCACCAG ACCTGCTGAA ACAGCCAACC CCCAGCACCT CCACCCAACC CCTCCACTCC TCAACAAACC CAAATGATGT GCCAAATAAG -756 ATAAAGGTGC CACCTGGCCC AGTTCTTTTT GTCCTAATGT AGCTCCCAGC ACTCCAAGCA ATTACAAGCA CAATACAGCA GATTCGGGT -666 PPAR $\gamma$

CACTGGGAGA TGAAAGACCA AAAATGTGGG CCTTAAGCGT TTATTAGGAA ACACTGTCCT TTAAGAAAAA AAAGTAGGCC TATGTAGTTG -576 HNF4a

TCTGGCTGTG AATCTGCCTT ACTTAGCCTA GTGAAAAAAC ATCAACTCTC ATACTAAAAG TTGCATTTCT CCTATCTGCA ACCCTTCTCT -486 STAT1
TCATTCTGAT CTACAGATAT AATATCTACA GTGGGCGTTT AaCTGTTGTA CATGTCACAA CAATTACTCA GCTGAGCTCC ATGATTTAAT -396
GATA

ATCCTCTTTC AATTAAATGT GGCCAAAAAC ATATCTTTCT AAACTTTCTG TGGCATTTTG TCACAAAAAT CCTAAAAAAG ATATAATAAT -306 GATA

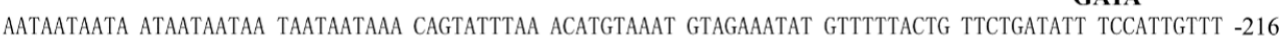

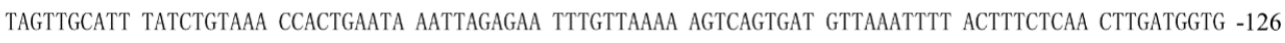
GATTATTTTC CTTTCAACAT GaTACCTATG TGTTTTATTC CTTACTTACA CAACAATACA TGCGAAACAT TACATCACAG ATGAAACCTC -36

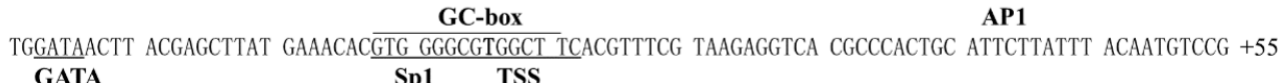
GATA
GACGTGTTTA ACCCCAAGCA ACTCTTTCAC CGCGCGTGGC ACAAACTCTT ACTTATTACT TAACGGTAAG ACAAGTAATA ATAACGATTT +145 AAATTGCTTT GCAAGTCATT AAACAGTTCC TTTTTTGCGT CCTTCTTTCA TAACCAAGCC +205

Figure 2. Nucleotide sequences and putative regulatory elements of the TSPO promoter in $P$. fulvidraco. Numbers are relative to the transcription start site $(+1)$. The putative regulatory elements are indicated in bold letters below the underlined sequence. 


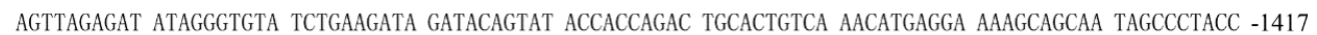
TCAGTAAAAT GTTATAGTAA GCAAAGTAAT AAAATTAGGT GACTCCTTTG GGTTCCTGGG CAGGAAGCTA AGCAAGCCCC CCACCTATCC -1327 AACTAAACAT ACTAATGCAT GGAGCCCTGA GTGCTCTGCT CTGCAACATA ATATGTAGAA AATAATTTTT TTTACTAAAT TACACTTTCA -1237 TTCTTAATAT TTGAGGAACC TCTGTGCTTT GCAATCTGTG AAGGCAATCT AATCTTATGC AAATGTCTGG TTTCTTTTTC ATTTTTAAAC -1147 ATTTTTTTCT TCTGCTCATT GATTACAAAT TAAGTCACAT ACACACTAGT CACATTTCTG AATGTAATTT AAATTCTGTG ACTCTTTACA -1057 TTTGTTATCC TATGTACGGg GTTGTGTGTG ACTGTTTTAG CCTAATCATT CACTTCAAAT CAAATCAAAT CAAATCAAAT TTTATTTGTC -967 ACATACACAT ACATACAGTA CAGgGTACGA TATGCAgTgA AATGCTTTAT GACACTGTCT GgTATAAGAA TAAAAAGAT ATATAAAAAG -877 TATATAAAAA GCAATAAATA AAATAAGAAT AAAAATAATA ACAAGTATAA ACTGTATAAG AAAACTATAT AAGAAAAACT ATATAAAAAT -787 ATGAAAATAT AGATGAAGAA ATAATGTATA TACATCTGCA TAATATGCAT ATACATAAAT GTGTATAGTA TTTGAAGATT GTCCTTAAAG -697 FOXL2

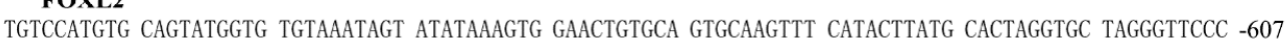

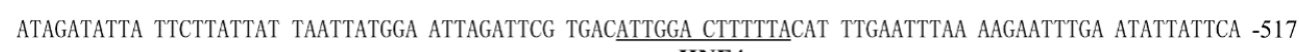
AATATTTATT CTGAAGTTGC ATGTAGTTGT GCCTCAGGCT AAAATGTCT ACTATACTAC CTGATGGGGA TAAAACAAAC AAACAAACAA -427 ACAACCCACA ATTAAACATG GTTAATACTA CTAACGATAT AATTAAAAAA GAAAAGAAAa GAAAAAGTCA CAGTCAGGAT TATGAaGGTT -337 CTGACTGCAA ACTGTTTTCA GTACACTAGG CTTCCACTGT ATGCTATCAG ATAGGTACAC TCTAGCTCAC ACAACCCAAC GTCTGGTTTT -247 DCE CTGTAGTAAG TATACTGTAC CATATTATTA GGTTTTTATG TCACGTGTGC GCTGAATCCT AATGAAATTT TGACCGAGAC AGACACCAAC -157 GTCACTGTTC ATTGTGATTC TGTGTAGTCT GTCTGGCTCA GAAGAGTGTA ATGATTGGCT GGATTCACTA TAATCCCCCT CCCTCCTTCC -67

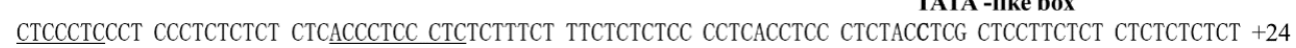
Sp1 Sp1 TSS CTCTCTCTCT стCTCTCTCT стCTCTCTCT CTCTCTCTGG GAGCGGCAGG ATGTGCGAAT GAGGG +89

Figure 3. Nucleotide sequences and putative regulatory elements of the SMAD4 promoter in P. fulvidraco. Numbers are relative to the transcription start site $(+1)$. The putative regulatory elements are indicated in bold letters below the underlined sequence.

\subsection{5'-Deletion Assay of the Regions of TSPO and SAMD4 Promoters}

The sequence deletion from $-1076 \mathrm{bp}$ to $-504 \mathrm{bp}$ of TSPO promoter significantly decreased the luciferase activity of the TSPO promoter. Subsequent absence from $-1558 \mathrm{bp}$ to $-1076 \mathrm{bp}$ significantly increased its luciferase activity and the sequence deletion from -2015 bp to -1558 bp showed no significant effect (Figure $4 \mathrm{~A}$ ). These results demonstrated that the -1076 to -504 bp sequence contained the positively regulatory elements for TSPO expression, and the -1558 to $-1076 \mathrm{bp}$ sequence contained the negatively regulatory elements for TSPO expression. For the SMAD4 promoter, the sequence deletion from $-999 \mathrm{bp}$ to $-559 \mathrm{bp}$ reduced the luciferase activity, whereas the sequence deletion between $-1506 \mathrm{bp}$ and $-999 \mathrm{bp}$ did not influence its luciferase activity (Figure 4B).

A

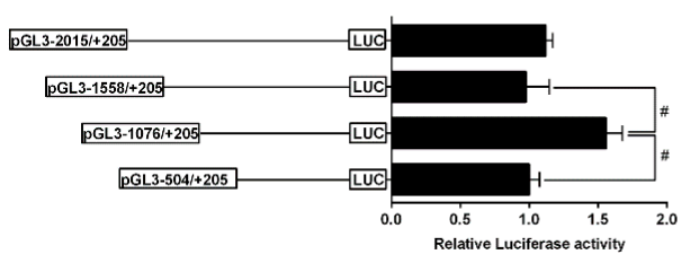

B

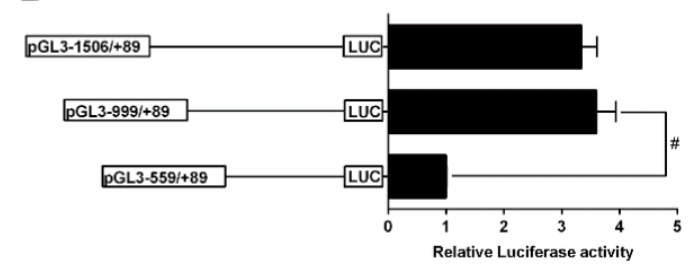

Figure 4. 5' unidirectional deletion analysis of the TSPO and SMAD4 promoters for yellow catfish. Schematic diagram of truncated promoters is shown at left panel. The corresponding luciferase reporter assay results are shown at right panel. A series of plasmids containing $5^{\prime}$ unidirectional deletions of the TSPO (pGL3 -2015/+205, -1558/+205, -1076/+205, $-504 /+205$ ) and SMAD4 (pGL3 - 1506/+89, -999/+89, -559/+89) promoter regions fused in frame to the luciferase gene were transfected into HEK293T cells. Values represent the ratio between firefly and Renilla luciferase activities, normalized to the control plasmid pGl3 $-504 /+205$ (A) and pGl3 -559/+89 (B), respectively. Results are shown as mean \pm standard error of mean (SEM) $(n=3)$. Hash symbol (\#) means significant differences between two groups $(p<0.05)$. 
Since SMAD4 promoter region has no putative PPAR $\gamma$ and STAT3 sites, we next only examined the transcriptional activity of TSPO with $5^{\prime}$-deletion mutants which was influenced by PPAR $\gamma$ and STAT3 overexpression on TSPO. Compared with the control, PPAR $\gamma$ overexpression up-regulated the promoter activity. The deletion from $-1076 \mathrm{bp}$ to $-504 \mathrm{bp}$ alleviated the PPAR $\gamma$ overexpression-induced TSPO promoter activity, and from $-2015 \mathrm{bp}$ to $-1558 \mathrm{bp}$ increased PPAR $\gamma$ overexpression-induced TSPO promoter activity, indicating that $-1076 /-504$ and $-2015 /-1558$ regions of TSPO promoter were affected by PPAR $\gamma$ overexpression (Figure 5A). On the contrary, the promoter activity was decreased under the STAT3 over-expression, and the TSPO promoter region from $-2015 \mathrm{bp}$ to $-1558 \mathrm{bp}$ further enhanced the inhibition of STAT3 overexpression. Nonetheless, the sequence deletion of TSPO promoter between $-1076 \mathrm{bp}$ and $+205 \mathrm{bp}$ region completely abolished the STAT3-induced down-regulation, reflecting that the negative response elements existed at $-2015 /-1558 \mathrm{bp}$ and $-1558 /-1076 \mathrm{bp}$ regions of TSPO promoter to STAT3 (Figure 5B).
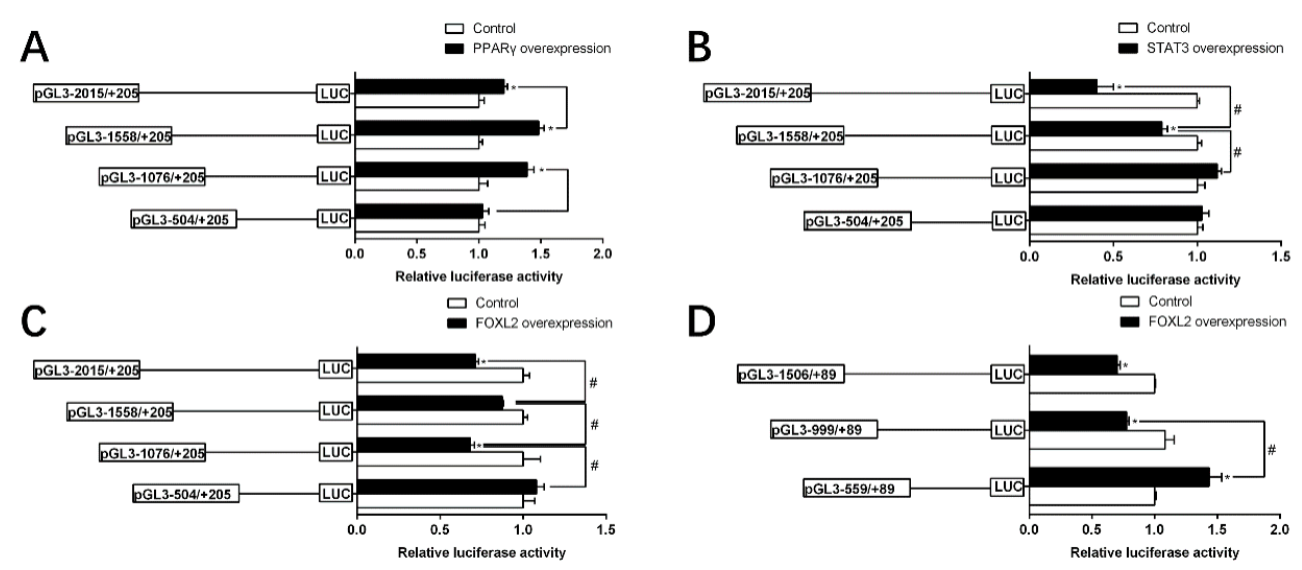

Figure 5. Overexpression analysis of $5^{\prime}$ unidirectional deletion assays of the TSPO and SMAD4 promoters of yellow catfish. (A) PPAR $\gamma$ overexpression; (B) STAT3 overexpression and (C,D) FOXL2 overexpression. Values represent the ratio between firefly and Renilla luciferase activities, normalized to the control. Results are shown as mean \pm standard error of mean (SEM) $(n=3)$. Hash symbol (\#) means significant differences between two groups $(p<0.05)$. Asterisk $\left(^{*}\right)$ indicate significant differences between different treatments with the same plasmid $(p<0.05)$.

The response of these promoters to FOXL2 overexpression was investigated (Figure 5C,D). For the TSPO promoter, the deletion plasmids of $-1076 /-504$ and $-2015 /-1558$ markedly alleviated the FOXL2 overexpression-induced TSPO promoter activity (Figure 5C). For the SMAD4 promoter, overexpression of FOXL2 markedly reduced the promoter activity compared to the control. The inhibitory effect by FOXL2 was completely abolished when the sequence between -999 and $-559 \mathrm{bp}$ was deleted, indicating that there are negative response elements at $-999 /-559$ bp region of SMAD4 promoter to FOXL2 (Figure 5D).

\subsection{Site-Mutation Analysis of Binding Sites on the Regions of TSPO and SMAD4 Promoters}

Next, we performed the site mutation to further elucidate whether TSPO and SMAD4 promoters possessed functional binding sites of PPAR $\gamma$, STAT3 and FOXL2 (Figure 6). The mutation of the $-734 /-748$ PPAR $\gamma$ binding site reduced the PPAR $\gamma$ overexpressioninduced luciferase activity significantly, demonstrating that this site positively mediated TSPO transcription (Figure 6A). Compared with the pGl3 -2019/+205 TSPO vector, the mutation of STAT3 binding site between the -1507 and -1516 significantly escalted the luciferase activity in the STAT3 overexpression group, indicating the STAT3 site inhibited STAT3 overexpression-induced TSPO transcription (Figure 6B). Overexpressed FOXL2 reduced TSPO promoter activity compared to the control, but the mutated FOXL2 abolished its inhibitory effect, demonstrating that FOXL2 site inhibited FOXL2-induced TSPO transcription (Figure 6C). And the mutation of the SMAD4-FOXL2 (-777/-789) site did 
not affect FOXL2-overexpression SMAD4 promoter activity, indicating that the $-1506 /+89$ region of SMAD4 did not possess the FOXL2 site (Figure 6D).
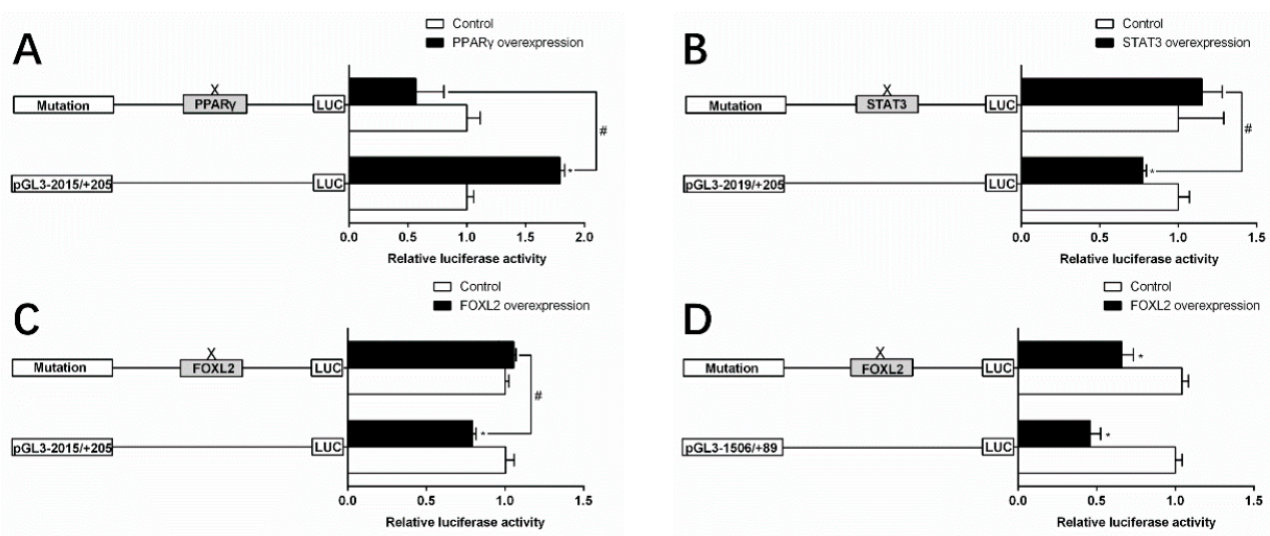

Figure 6. Assays of predicted PPAR $\gamma$, STAT3 and FOXL2 binding sites after site-directed mutagenesis. (A) Site mutagenesis of PPAR $\gamma$ on $-2015 /+205$ TSPO promoter. (B) Site mutagenesis of STAT3 on -2015/+205 TSPO promoter. (C) Site mutagenesis of FOXL2 on -2015/+205 TSPO promoter. (D) Site mutagenesis of FOXL2 on $-1506 /+89$ SMAD4 promoter. Values are presented as mean $\pm \operatorname{SEM}(n=3)$. Hash symbol (\#) means significant differences between two groups $(p<0.05)$. Asterisk $\left(^{*}\right)$ indicate significant differences between different treatments with the same plasmid $(p<0.05)$.

2.6. EMSA for the Confirmation of the Functional Binding of PPAR $\gamma$, STAT3 and FOXL2 on the TSPO and SMAD4 Promoters

EMSA was performed to demonstrate whether these putative binding sites could interact with PPAR $\gamma$, STAT3, and FOXL2. For the TSPO promoter, the 300-fold unlabeled PPAR $\gamma$ binding sites ( $-734 \mathrm{bp} /-748 \mathrm{bp}$ ) did not compete with the PPAR $\gamma$ binding sequence as the probe, indicating that PPAR $\gamma$ could not bind with this region (Figure 7A). However, the 300-fold unlabelled STAT3 and FOXL2 binding sites competed for the binding with the STAT3 and FOXL2 binding sequences as the probe, respectively, and reduced the brightness of the labeled probe, and the 300-fold unlabeled mutated STAT3 and mutated FOXL2 binding region declined this competition, implying that TSPO could be bound by STAT3 (Figure 7B) and FOXL2 (Figure 7C).

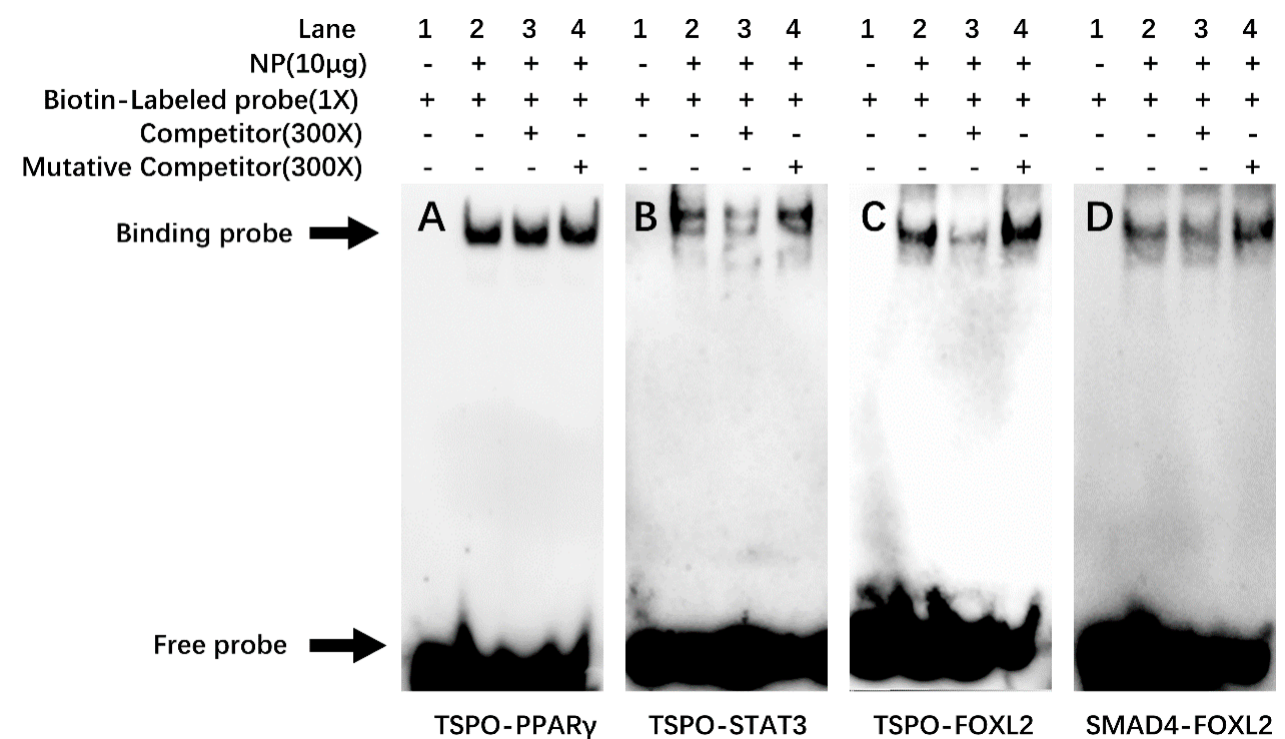

Figure 7. EMSA analysis of predicted SREs. (A) -734/-748 binding site of TSPO (TSPO-PPAR $\gamma$ ); (B) $-1507 /-1516$ binding site of TSPO (TSPO-STAT3); (C) -1650/-1663 binding site of TSPO (TSPOFOXL2); (D) -777/ -789 binding site of SAMD4 (SAMD4- FOXL2). 
For SMAD4 promoter, when the FOXL2 binding sequence was used as the probe, the 300-fold unlabeled FOXL2 binding site ( $-777 \mathrm{bp} /-789 \mathrm{bp})$ did not compete for the binding, demonstrating that FOXL2 could not bind to this region (Figure 7D).

\section{Discussion}

TSPO and SMAD4 play an important role in steroid synthesis [1,3], but the current research on these two genes mainly focuses on mitochondrial function [14], oxidative stress [15] and signal transduction [16]. Several studies reported that TSPO ligand promoted pregnenolone synthesis [14], and TGF- $\beta$ /SMAD4 signaling pathway regulated steroid production and ovarian development [17], but these studies are rare in fish. Considering that the characterization of gene sequence is helpful to study their function, we identified the cDNA sequences of TSPO and SMAD4 and explored their mRNA tissue expression from yellow catfish. We also characterized the TSPO and SMAD4 promoters. Our research laid a foundation for further investigation into their function.

Our study found that the protein sequences of $P$. fulvidraco TSPO had similar domains with mammals, such as five $\alpha$-helix transmembrane structure (TM1-5), the conserved CRAC domain in the C-terminus, in agreement with other studies $[18,19]$. These indicated that the TSPO was highly evolutionarily conserved, as reported by Jaremko et al. [20]. Our study also demonstrated that the SMAD4 protein possessed six domains, such as Nterminal MH1 domain, C-terminal MH1 domain, SAD domain, DNA Binding motif, NLS domain and NES domain, similar to several studies [21,22]. Studies suggested that these domains were essential for their effector functions responsible for active nucleocytoplasmic shuttling of SMAD4 [23,24].

In this study, we demonstrated the tissue distribution of these genes, which provided the basis for elucidating their functions. Our results indicated that their mRNAs were existent in ten tissues in the P. fulvidraco, indicating that steroid synthetase plays a wide role in these tissues. Similarly, studies suggested the major sites of steroidogenesis included several gonadal and non-gonadal tissues, such as head kidney, intestine, liver and adipose tissue $[25,26]$. Our study indicated that TSPO mRNA levels were the highest in the spleen, followed by the testis, kidney and muscle, while there was no significant difference in heart, liver, brain, fat, intestine and ovary. In zebrafish, Rampon et al. [27] reported that TSPO mRNA expression was the highest in the heart, followed by the spinal cord, muscle, ovary, testis and intestine, the lowest in the brain, indicating species-specific differences. In addition, our study indicated that SMAD4 mRNA amounts were predominant in the testis, followed by the liver and ovary, and the lowest in the kidney. In gooses, suggested that SMAD4 mRNAs were expressed in the ovary, hypothalamus and pituitary [28]. The high expression of SAMD4 in the ovary may be related to the secretion of sex hormones. SAMD4, as a transcription factor, can regulate the synthesis of FSH, thus affecting the development of gonads [29]. Their distinctive tissue distribution in P. fulvidraco revealed the functional differentiation of these proteins and reflected the tissue-specific metabolic regulation.

In eukaryotes, the identification of the core regions of promoters is crucial for exploring the mechanism of transcriptional initiation [30]. In the present study, we found Sp1 binding sites in the core region of TSPO promoter. Studies suggested that SP transcriptional sites are important for the basal activity of TSPO promoters [31]. Batarseh et al. [6] found several GC-rich sequences in the proximal region of the TSPO promoter in mouse, in agreement with the present study. Wierstra [32] reported that SP1 directly bound with GC-rich domains and modulated transcription after various stimuli. Thus, we speculated that SP1-rich and GC-rich regions positively regulated TSPO promoter activity. However, we found that this region of SMAD4 promoter had no typical promoter characteristics, such as the high CG content or TATA-box, but had some TATA-like structures (TATAAT) and other potential binding sites, as in other reports [33]. Studies have shown that the promoter lacking TATA possessed various Sp1 binding sites [34]. We also found three Sp1 sites and a downstream core element (DCE) in the core SMAD4 promoter region. These transcription factors ensure the normal initiation of gene transcription [35]. 
The identification of transcription factor binding sites (TFBS) is significant to reveal the regulatory mechanism of genes [36]. Our study found some binding sites, such as SOX, SRY, GATA, PPAR $\gamma$, STAT1, Pu.1, AP1, FOXL2 and STAT3 in the promoter region of TSPO, which are similar to other reports in mice [37], reflecting that TSPO participated in many physiological progresses. Rashid et al. [10] pointed out that TSPO was transcriptionally regulated by these transcription factors such as Sp1, Pu.1 and AP1. For SMAD4 promoter, we found Ap1, HNF4 $\alpha$, ZFP and FOXL2 in the SMAD4 promoter region. However, different from human, there are other transcription factors such as F2F, Pit-1 and AP1 in SMAD4 promoters [9]. These indicated that the transcriptional regulation of SMAD4 was complex because maybe multiple transcription factors mediated its regulation, thus playing an important role in fish.

PPAR $\gamma$, STAT3 and FOXL2 regulate the transcription of key enzymes in steroidogenesis $[13,38]$. The present study found that PPAR $\gamma$ significantly increased the transcriptional activity of TSPO promoter, while STAT3 and FOXL2 decreased TSPO transcription. These results indicated that PPAR $\gamma$, STAT3 and FOXL2 differentially regulated steroidogenesis by targeting the TSPO in yellow catfish. However, we identified a functional binding site of STAT3 and FOXL2 in this region, but not PPAR $\gamma$, after the further site mutagenesis and EMSA. Similarly, Batarseh et al. [39] reported that there was a STAT3 binding site in the TSPO promoter. For the SMAD4 promoter, although FOXL2 overexpression affected the promoter activity, site mutagenesis and EMSA identified SMAD4 is not the target gene of FOXL2. Both SMAD4 and FOXL2 are transcription factors of CYP19A1 [3,38], but FOXL2 didn't regulate SMAD4. Thus, it is possible that the SMAD4 promoter has other steroidal synthesis regulatory factors.

In summary, we cloned the cDNA sequences of TSPO and SMAD4 genes from yellow catfish, explored their mRNA tissue distribution, and characterized the TSPO and SMAD4 promoter regions. The promoters of TSPO and SMAD4 genes presented different structures on their core regions. The present study demonstrated that TSPO was the direct target gene of STAT3 and FOXL2. These studies are of great significance to demonstrate transcriptional regulation of key genes of steroid synthase in vertebrates.

\section{Materials and Methods}

\subsection{Ethical Statement and Experimental Procedures}

All animal experiments followed the Institutional Ethical Guidelines of Huazhong Agricultural University (HZAU) for using the vertebrates and were approved by the Experimentation Ethics Committee of our university (Wuhan, Hubei, China) (ID Code: Fish-2018-0827, Date: 27 August 2018).

The present study included two experiments. We cloned TSPO and SMAD4 genes, and explored their mRNA tissue distribution in Expt. 1, characterized the upstream $5^{\prime}$ flanking regions of the TSPO and SMAD4 genes and explored their promoter activity by the deletion and mutation analysis in Expt. 2.

\subsection{Experimental Animals and Reagents}

Juvenile yellow catfish $(22.5 \pm 3.1 \mathrm{~g}$, mean $\pm \mathrm{SEM})$ for cDNA and promoter cloning were purchased from a commercial farm (Wuhan, China). They were maintained in indoor cylindrical fiberglass tanks (300-L water volume) at ambient temperature for 2-week acclimation. All fish were fed a commercial pellet diet twice a day and provided with continuous aeration to maintain the dissolved oxygen level near saturation. At the end of 2-week acclimation, fish were fasted for $24 \mathrm{~h}$ and then euthanized with MS-222 (100 $\mathrm{mg} / \mathrm{L}$ ). The heart, brain, liver, kidney, muscle, spleen, fat, intestine, testis and ovary were sampled and stored at $-80^{\circ} \mathrm{C}$ for the subsequent analysis. The HEK293T cell line was from Cell Resource Center in Fishery College, Huazhong Agricultural University. Dulbecco's Modified Eagle's medium (DMEM), fetal bovine serum (FBS) and $0.25 \%$ trypsin-EDTA were from Gibco (Waltham, MA, USA). Other reagents were from Sigma-Aldrich (Saint 
Louis, MO, USA). Using ClonExpress II One Step Cloning Kit (Vazyme, Piscataway, NJ, USA), the overexpression plasmid was labeled with pcDNA3.1 (+) vector.

\subsection{Expt. 1: Cloning and Tissue Distribution of Gene Expression}

\subsubsection{RNA Isolation, cDNA Cloning and Sequence Editing}

RNA isolation and cDNA cloning of TSPO and SMAD4 followed the protocol described in our previous study [40]. The $3^{\prime}$ - and $5^{\prime}$-end sequences were obtained through the nested $3^{\prime}$ and $5^{\prime}$ RACE PCR via the SMART RACE cDNA Amplification Kit (Clontech, USA). The primers were shown in Table S1. The full-length cDNA sequences of TSPO and SMAD4 were edited by EDITSEQ (DNA star) to find the open reading frame (ORF). They were then translated into the amino acid sequence by standard genetic code. Cluster-w multiple alignment algorithm was used to evaluate the sequence alignment and amino acid conservation. On March 5, 2019, NCBI's online CDD tool (http:/ / www.ncbi.nlm.nih.gov/Structure/cdd/wrpsb.cgi (accessed on 20 April 2021)) was used to analyze the domains.

\subsubsection{Real-Time Quantitative PCR (qPCR)}

qPCR method [41] was used to determine the mRNA expression. The primer sequences of genes for qPCR analysis are given in Table S2. The ten housekeeping genes ( $\beta$-actin, 18s rrna, rpl7, gapdh, hprt, tbp, elfa, tuba and ubce) were selected to analyze their transcriptional stability. The relative expression of genes was calculated via the $2^{-\Delta \Delta \mathrm{Ct}}$ method after normalizing to the geometric mean after the best combination of two genes, based on geNorm analysis. Prior to the analysis, we performed experiments to check the stability of housekeeping genes, and $\beta$-actin and ubce $(M=1.2669)$ presented the most stable expression level for analyzing the tissue distribution.

\subsection{Expt. 2: Structure and Functional Analysis of Promoter}

\subsubsection{Cloning of Promoter and Plasmid Construction}

Via RNA ligase-mediated rapid amplification of $5^{\prime}$ cDNA ends (RLM-5' RACE) method, we identified the $5^{\prime}$ cDNA sequences and the transcription start sites (TSS) of TSPO and SMAD4. We cloned the promoter sequence, based on the published draft genome of yellow catfish [42] and our Expt. 1 above, and the protocols were similar to those in our recent studies [43]. Genomic DNA was extracted from yellow catfish tail fins using the commercial kit (Omega, USA). In order to amplify the TSPO and SMAD4 promoter sequences, we designed the specific primers with (Sac I and Hind III) restriction sites (Table S3). To generate the luciferase reporter constructs, we purified the PCR product and pGl3-Basic vectors (Promega, USA) and digested them with corresponding endonucleases, and then these products were ligated via ClonExpress II One Step Cloning Kit (Vazyme, Piscataway, NJ, USA). According to the distance from their TSS, we named the plasmids as pGl3 $-2015 /+205$ of TSPO vector and pGl3 $-1506 /+89$ of SMAD4 vector, respectively. Using the template of pGl3 $-2015 /+205$ of TSPO vector, we produced the plasmids pGl3 $-1558 /+205$, pGl3 $-1076 /+205$ and pGl3 $-504 /+205$ of TSPO vector. Similarly, using pGl3 $-1506 /+89$ of SMAD4 vector as a template, we generated plasmids pGl3 $-999 /+89$ and $\mathrm{pGl} 3-559 /+89$.

\subsubsection{Sequence Analysis}

For sequences analysis of the TSPO and SMAD4 promoters in yellow catfish, their TFBSs were predicted by the online tools on August 9, 2020 (http:/ /www.genomatix.de/ and http://jaspar.genereg.net/ (accessed on 20 April 2021)). We used the Clustal-W multiple alignment to evaluate sequence alignments.

\subsubsection{Transfections and Luciferase Assays}

We transiently transfected the plasmid into HEK293T cells with Lipofectamine 2000 (Invitrogen, Carlsbad, CA, USA), based on the manufacturer's instruction. The reporter 
plasmids were used in equimolar amounts in Opti-MEM (Invitrogen, Carlsbad, CA, USA). They were co-transfected with $20 \mathrm{ng}$ pRL-TK as a control. After 4-h transfection, the medium was replaced by DMEM plus $10 \%$ FBS. Then, after 24-h incubation, we harvested cells to determine the luciferase activity by Dual-Luciferase Reporter Assay System (Promega, Madison, WI, USA) after calculating the ratio of firefly luciferase to Renilla luciferase. These experiments were performed in triplicates.

To explore the overexpression-induced changes of promoter activities, we co-transfected the overexpression plasmid or the same amount of pcDNA3.1 (+) plasmid (control) with the TSPO and SMAD4 luciferase reporter plasmids into HEK293T cells using Lipofectamine 2000 (Invitrogen, Carlsbad, CA, USA). The remaining steps are the same as above.

\subsubsection{Site-Mutation Analysis of Binding Sites on the TSPO and SMAD4 Promoters}

To identify the corresponding PPAR $\gamma$, STAT3 and FOXL2 binding sites on the yellow catfish TSPO and SMAD4 promoters, we performed the site-directed mutagenesis, based on the instruction of QuickChange II Site-Directed Mutagenesis Kit (Vazyme, Piscataway, NJ, USA). The pGl3-TSPO-2015 and pGl3-SMAD4-1558 were used as the template, respectively. The mutagenesis primers were presented in Table S3. These mutant constructs were named as Mutation-PPAR $\gamma$, Mutation-STAT3 and Mutation-FOXL2, respectively. To study the overexpression-induced changes of PPAR $\gamma$, STAT3 and FOXL2 binding sites, we cotransfected overexpression plasmid (300 ng) or pcDNA3.1 (+) plasmid (control, $300 \mathrm{ng}$ ) with wild-type plasmid ( $500 \mathrm{ng}$ ) or mutation plasmid (500 ng) into HEK293T cells by Lipofectamine 2000 (Invitrogen, Carlsbad, CA, USA). The remaining steps are the same as above.

\subsubsection{Electrophoretic Mobility-Shift Assay (EMSA)}

EMSA was performed to analyze the PPAR $\gamma$, STAT3 and FOXL2 functional binding sites on the regions of TSPO and SMAD4 promoters after Xu et al. [43]. Cytoplasmic and nuclear extracts were obtained with the same protocols of $\mathrm{Xu}$ et al. [43]. We extracted the nuclear proteins from HEK293T cells, and used the bicinchoninic acid assay (BCA) to determine the protein content. Table S4 listed the oligonucleotide sequences for EMSA.

\subsection{Statistical Analysis}

The data were showed as means \pm standard error of mean (SEM). Before the statistical analysis, all data were tested for distribution normality using the Kolmogornov-Smirnov test. Using the unpaired two-tailed Student's $t$ test, we determined the differences between two groups. Difference was thought to be statistically significant at $p<0.05$. The SPSS 19.0 for Windows (SPSS Chicago, IL, USA) was used for the statistical analyses.

Supplementary Materials: The following are available online at https:/ / www.mdpi.com/article/10 .3390 /ijms22094505/s1, Table S1: Nucleotide sequences of the primers used for the cDNA cloning from P. fulvidraco, Table S2: Primers used for real-time quantitative PCR analysis, Table S3: Primers used for cloning and functional analysis of promoters in the experiments, Table S4: Primers used for electrophoretic mobility-shift assay. Figure S1: Multiple amino acid sequence alignment of TSPO from P. fulvidraco and other species, Figure S2: Multiple amino acid sequence alignment of SMAD4 from $P$. fulvidraco and other species.

Author Contributions: F.C.: Investigation, Methodology, Writing-original draft; C.-C.Z. and C.-C.S.: Methodology, Writing - review and editing. S.-W.C. and Y.H.: Methodology, Writing-review and editing. X.-Y.T.: Funding acquisition, Project administration, Supervision, Writing-review and editing. All authors have read and agreed to the published version of the manuscript.

Funding: This work was supported by National Natural Science Foundation of China (grant No.: 31572605) and by the National Key R\&D Program of China (grant No. 2018YFD0900400).

Institutional Review Board Statement: All animal experiments followed the Institutional Ethical Guidelines of Huazhong Agricultural University (HZAU) for using the vertebrates and were approved by the Experimentation Ethics Committee of our university (Wuhan, Hubei, China) (ID Code: Fish-2018-0827, Date: 27 August 2018). 
Informed Consent Statement: Not applicable.

Data Availability Statement: Data is contained within the article or Supplementary Materials.

Acknowledgments: We thank all the staffs in Laboratory of Molecular Nutrition for Aquatic Economic Animals, Fishery College, Huazhong Agricultural University, for their help during the experiments.

Conflicts of Interest: The authors disclosed no conflict of interest.

\begin{abstract}
Abbreviations
TSPO, translocator protein; STAR, steroidogenic acute regulatory protein; SMAD4, mothers against decapentaplegic homolog 4; SAD, SMAD4 activation domain; NLS, nuclear localization signal; NES, nuclear export signal; DMEM, Dulbecco's Modified Eagles Medium; FBS, fetal bovine serum; PBS, phosphate-buffer saline; TFBS, transcriptional factor binding sites; TSS, transcription start site; Sp, specificity protein; AP1, activator protein-1; HNF4 $\alpha$, hepatic nuclear factor $4 \alpha$; PPAR $\gamma$, peroxisome proliferator-activated receptor $\gamma$; Pu.1, PU box binding-1; STAT, signal transducer and activator of transcription; FOXL2, forkhead box 12; SOX1, SRY-box transcription factor 1; SRY, sex determining region $\mathrm{Y}$
\end{abstract}

\title{
References
}

1. Lacapère, J.-J.; Papadopoulos, V. Peripheral-Type Benzodiazepine Receptor: Structure and Function of a Cholesterol-Binding Protein in Steroid and Bile Acid Biosynthesis. Steroids 2003, 68, 569-585. [CrossRef]

2. Li, J.; Daly, E.; Campioli, E.; Wabitsch, M.; Papadopoulos, V. De Novo Synthesis of Steroids and Oxysterols in Adipocytes. J. Biol. Chem. 2014, 289, 747-764. [CrossRef] [PubMed]

3. Li, Q.; Du, X.; Pan, Z.; Zhang, L.; Li, Q. The Transcription Factor SMAD4 and miR-10b Contribute to E2 Release and Cell Apoptosis in Ovarian Granulosa Cells by Targeting CYP19A1. Mol. Cell. Endocrinol. 2018, 476, 84-95. [CrossRef] [PubMed]

4. $\quad$ Rashid, H.; Kitano, H.; Lee, K.H.; Nii, S.; Shigematsu, T.; Kadomura, K.; Yamaguchi, A.; Matsuyama, M. Fugu (Takifugu Rubripes) Sexual Differentiation: CYP19 Regulation and Aromatase Inhibitor Induced Testicular Development. Sex. Dev. 2007, 1, 311-322. [CrossRef] [PubMed]

5. Yoo, S.-Y.; Lee, J.-A.; Shin, Y.; Cho, N.-Y.; Bae, J.M.; Kang, G.H. Clinicopathological Characterization and Prognostic Implication of SMAD4 Expression in Colorectal Carcinoma. J. Pathol. Transl. Med. 2019, 53, 289-297. [CrossRef]

6. Batarseh, A.; Barlow, K.D.; Martinez-Arguelles, D.B.; Papadopoulos, V. Functional Characterization of the Human Translocator Protein (18kDa) Gene Promoter in Human Breast Cancer Cell Lines. Biochim. Biophys. Acta Bioenerg. 2012, 1819, 38-56. [CrossRef]

7. Nikolic, A.; Ristanovic, M.; Zivaljevic, V.; Rankov, A.D.; Radojkovic, D.; Paunovic, I. SMAD4 Gene Promoter Mutations in Patients with Thyroid Tumors. Exp. Mol. Pathol. 2015, 99, 100-103. [CrossRef]

8. Chen, S.-W.; Wu, K.; Lv, W.-H.; Chen, F.; Song, C.-C.; Luo, Z. Functional Analysis of Two Zinc (Zn) Transporters (ZIP3 and ZIP8) Promoters and Their Distinct Response to MTF1 and RREB1 in the Regulation of Zn Metabolism. Int. J. Mol. Sci. 2020, $21,6135$. [CrossRef]

9. Minami, R.; Kitazawa, R.; Maeda, S.; Kitazawa, S. Analysis of 5'-Flanking Region of Human Smad4 (DPC4) Gene. Biochim. Biophys. Acta Gene Struct. Expr. 1998, 1443, 182-185. [CrossRef]

10. Rashid, K.; Geissl, L.; Wolf, A.; Karlstetter, M.; Langmann, T. Transcriptional Regulation of Translocator Protein (18 kDa) (TSPO) in Microglia Requires Pu.1, Ap1 and Sp Factors. Biochim. Biophys. Acta Gene Regul. Mech. 2018, 1861, 1119-1133. [CrossRef]

11. Puttabyatappa, M.; Vandevoort, C.A.; Chaffin, C.L. hCG-Induced Down-Regulation of PPAR $\gamma$ and Liver X Receptors Promotes Periovulatory Progesterone Synthesis by Macaque Granulosa Cells. Endocrinology 2010, 151, 5865-5872. [CrossRef]

12. Wang, D.-S.; Kobayashi, T.; Zhou, L.-Y.; Paul-Prasanth, B.; Ijiri, S.; Sakai, F.; Okubo, K.; Morohashi, K.-I.; Nagahama, Y. Foxl2 Up-Regulates Aromatase Gene Transcription in a Female-Specific Manner by Binding to the Promoter as Well as Interacting with Ad4 Binding Protein/Steroidogenic Factor 1. Mol. Endocrinol. 2007, 21, 712-725. [CrossRef]

13. Lv, W.-H.; Chen, G.-H.; Zhuo, M.-Q.; Xu, Y.-H.; Xu, Y.-C.; Tan, X.-Y. Functional Analysis of Steroidogenic Factor 1 (sf-1) and $17 \alpha$-Hydroxylase/Lyase (cyp17 $\alpha$ ) Promoters in Yellow Catfish Pelteobagrus fulvidraco. Int. J. Mol. Sci. 2020, 22, 195. [CrossRef]

14. Lejri, I.; Grimm, A.; Hallé, F.; Abarghaz, M.; Klein, C.; Maitre, M.; Schmitt, M.; Bourguignon, J.-J.; Mensah-Nyagan, A.G.; Bihel, F.; et al. TSPO Ligands Boost Mitochondrial Function and Pregnenolone Synthesis. J. Alzheimer's Dis. 2019, 72, 1045-1058. [CrossRef]

15. Zeno, S.; Veenman, L.; Katz, Y.; Bode, J.; Gavish, M.; Zaaroor, M. The 18 kDa Mitochondrial Translocator Protein (TSPO) Prevents Accumulation of Protoporphyrin IX. Involvement of Reactive Oxygen Species (ROS). Curr. Mol. Med. 2012, $12,494-501$.

16. McCarthy, A.J.; Chetty, R. SMAD4/DPC4. J. Clin. Pathol. 2018, 71, 661-664. [CrossRef]

17. Pangas, S.A.; Li, X.; Robertson, E.J.; Matzuk, M.M. Premature Luteinization and Cumulus Cell Defects in OvarianSpecificSmad4Knockout Mice. Mol. Endocrinol. 2006, 20, 1406-1422. [CrossRef]

18. Iatmanen-Harbi, S.; Senicourt, L.; Papadopoulos, V.; Lequin, O.; Lacapere, J.-J. Characterization of the High-Affinity Drug Ligand Binding Site of Mouse Recombinant TSPO. Int. J. Mol. Sci. 2019, 20, 1444. [CrossRef] 
19. Selvaraj, V.; Stocco, D.M. The Changing Landscape in Translocator Protein (TSPO) Function. Trends Endocrinol. Metab. 2015, 26, 341-348. [CrossRef]

20. Jaremko, Ł.; Jaremko, M.; Giller, K.; Becker, S.; Zweckstetter, M. Conformational Flexibility in the Transmembrane Protein TSPO. Chemistry 2015, 21, 16555-16563. [CrossRef]

21. Barnes, E.; Askham, J.M.; Jones, P.F. cDNA Cloning and Molecular Characterization of Mink SMAD4. DNA Seq. 2002, 13, 47-53. [CrossRef] [PubMed]

22. Osman, A.; Niles, E.G.; LoVerde, P.T. Expression of Functional Schistosoma Mansoni SMAD4: Role in ERK-Mediated Transforming Growth Factor Beta (TGF-Beta) Down-Regulation. J. Biol. Chem. 2004, 279, 6474-6486. [CrossRef] [PubMed]

23. Inman, G.J.; Nicolás, F.J.; Hill, C.S. Nucleocytoplasmic Shuttling of SMADs 2, 3, and 4 Permits Sensing of TGF-Beta Receptor Activity. Mol. Cell 2002, 10, 283-294. [CrossRef]

24. Reguly, T.; Wrana, J.L. In or Out? The Dynamics of Smad Nucleocytoplasmic Shuttling. Trends Cell Biol. 2003, 13, 216-220. [CrossRef]

25. Rasheeda, M.K.; Kagawa, H.; Kirubagaran, R.; Dutta-Gupta, A.; Senthilkumaran, B. Cloning, Expression and Enzyme Activity Analysis of Testicular 11beta-Hydroxysteroid Dehydrogenase during Seasonal Cycle and after hCG Induction in Air-Breathing Catfish Clarias Gariepinus. J. Steroid Biochem. Mol. Biol. 2010, 120, 1-10. [CrossRef]

26. Swart, A.C.; Schloms, L.; Storbeck, K.-H.; Bloem, L.M.; Du Toit, T.; Quanson, J.L.; Rainey, W.E.; Swart, P. 11 $\beta$-Hydroxyandrostene dione, the Product of Androstenedione Metabolism in the Adrenal, is Metabolized in LNCaP Cells by $5 \alpha$-Reductase Yielding 11ß-Hydroxy-5 $\alpha$-Androstanedione. J. Steroid Biochem. Mol. Biol. 2013, 138, 132-142. [CrossRef]

27. Rampon, C.; Rouzaffour, M.; Ostuni, M.; Dufourcq, P.; Girard, C.; Freyssinet, J.M.; Lacapere, J.-J.; Schweizer-Groyer, G.; Vriz, S. Translocator Protein (18 kDa) is Involved in Primitive Erythropoiesis in Zebrafish. FASEB J. 2009, 23, 4181-4192. [CrossRef]

28. Huang, Z.; Yuan, X.; Wang, M.; Wu, N.; Song, Y.; Chen, Y.; Zhang, Y.; Xu, Q.; Chen, G.; Zhao, W. Molecular Cloning of the SMAD4 Gene and its mRNA Expression Analysis in Ovarian Follicles of the Yangzhou Goose (Anser Cygnoides). Br. Poult. Sci. 2016, 57, 515-521. [CrossRef]

29. Fortin, J.; Boehm, U.; Deng, C.; Treier, M.; Bernard, D.J. Follicle-Stimulating Hormone Synthesis and Fertility Depend on SMAD4 and FOXL2. FASEB J. 2014, 28, 3396-3410. [CrossRef]

30. Goodrich, J.A.; Tjian, R. Unexpected Roles for Core Promoter Recognition Factors in Cell-Type-Specific Transcription and Gene Regulation. Nat. Rev. Genet. 2010, 11, 549-558. [CrossRef]

31. Shimoyama, S.; Furukawa, T.; Ogata, Y.; Nikaido, Y.; Koga, K.; Sakamoto, Y.; Ueno, S.; Nakamura, K. Lipopolysaccharide induces Mouse Translocator Protein (18 kDa) Expression via the AP-1 Complex in the Microglial Cell Line, BV-2. PLoS ONE 2019, 14, e0222861. [CrossRef]

32. Wierstra, I. Sp1: Emerging Roles-Beyond Constitutive Activation of TATA-Less Housekeeping Genes. Biochem. Biophys. Res. Commun. 2008, 372, 1-13. [CrossRef]

33. Calva, D.; Dahdaleh, F.S.; Woodfield, G.; Weigel, R.J.; Carr, J.C.; Chinnathambi, S.; Howe, J.R. Discovery of SMAD4 Promoters, Transcription Factor Binding Sites and Deletions in Juvenile Polyposis Patients. Nucleic Acids Res. 2011, 39, 5369-5378. [CrossRef]

34. Smale, S.T.; Kadonaga, J.T. The RNA Polymerase II Core Promoter. Annu. Rev. Biochem. 2003, 72, 449-479. [CrossRef]

35. Lenhard, B.; Sandelin, A.; Carninci, P. Metazoan Promoters: Emerging Characteristics and Insights into Transcriptional Regulation. Nat. Rev. Genet. 2012, 13, 233-245. [CrossRef]

36. Chan, T.-M.; Leung, K.-S.; Lee, K.-H. TFBS Identification based on Genetic Algorithm with Combined Representations and Adaptive Post-Processing. Bioinformatics 2007, 24, 341-349. [CrossRef]

37. Giatzakis, C.; Papadopoulos, V. Differential Utilization of the Promoter of Peripheral-Type Benzodiazepine Receptor by Steroidogenic Versus Nonsteroidogenic Cell Lines and the Role of Sp1 and Sp3 in the Regulation of Basal Activity. Endocrinology 2004, 145, 1113-1123. [CrossRef]

38. Sridevi, P.; Chaitanya, R.; Dutta-Gupta, A.; Senthilkumaran, B. FTZ-F1 and FOXL2 Up-Regulate Catfish Brain Aromatase Gene Transcription by Specific Binding to the Promoter Motifs. Biochim. Biophys. Acta Bioenerg. 2012, 1819, 57-66. [CrossRef]

39. Batarseh, A.; Li, J.; Papadopoulos, V. Protein Kinase C Epsilon Regulation of Translocator Protein (18 kDa) TSPO Gene Expression is Mediated through a MAPK Pathway Targeting STAT3 and c-Jun Transcription Factors. Biochemistry 2010, 49, 4766-4778. [CrossRef]

40. Wei, C.-C.; Luo, Z.; Song, Y.-F.; Pan, Y.-X.; Wu, K.; You, W.-J. Identification of Autophagy related Genes LC3 and ATG4 from Yellow Catfish Pelteobagrus Fulvidraco and their Transcriptional Responses to Waterborne and Dietborne Zinc Exposure. Chemosphere 2017, 175, 228-238. [CrossRef]

41. Chen, Q.-L.; Luo, Z.; Wu, K.; Huang, C.; Zhuo, M.-Q.; Song, Y.-F.; Hu, W. Differential Effects of Dietary Copper Deficiency and Excess on Lipid Metabolism in Yellow Catfish Pelteobagrus Fulvidraco. Comp. Biochem. Physiol. Part B Biochem. Mol. Biol. 2015, 184, 19-28. [CrossRef] [PubMed]

42. Gong, G.; Dan, C.; Chen, N.; Gui, J.-F.; Mei, J.; Xiao, S.; Guo, W.; Huang, P.; Xiong, Y.; Wu, J.; et al. Chromosomal-Level Assembly of Yellow Catfish Genome using Third-Generation DNA Sequencing and Hi-C Analysis. GigaScience 2018, 7. [CrossRef] [PubMed]

43. Xu, Y.-H.; Luo, Z.; Wu, K.; Fan, Y.-F.; You, W.-J.; Zhang, L.-H. Structure and Functional Analysis of Promoters from Two Liver Isoforms of CPT I in Grass Carp Ctenopharyngodon idella. Int. J. Mol. Sci. 2017, 18, 2405. [CrossRef] [PubMed] 